\title{
Ipsilateral proprioceptive neuromuscular facilitation patterns improve overflow and reduce foot drop in patients with demyelinating polyneuropathy
}

\author{
Carolina S. Nakada', Paula C. Meningroni' ${ }^{1}$, Ana Claudia Silva Ferreira', Luciana Hata', Amanda C. Fuzaro', Wilson Marques Júnior², \\ João Eduardo de Araujo ${ }^{1, *}$ \\ 'Laboratory of Neuropsychobiology and Motor Behavior, Department of Health Sciences, Ribeirão Preto Medical School of the University of São Paulo (USP), Ribeirão Preto, \\ Brazil \\ ${ }^{2}$ Department of Neuroscience and Behavioral Sciences, Ribeirão Preto Medical School of the University of São Paulo (USP), Ribeirão Preto, Brazil
}

The purpose of this study was to evaluate the response of the tibialis anterior muscle (TAm) using surface electromyography in patients with Charcot-Marie-Tooth disease (CMT-IA), after ipsilateral proprioceptive neuromuscular patterns (PNF). Thirteen CMT-IA patients (both sexes) were treated twice a week, for 5 weeks, with bilateral PNF pattern, four times per treatment. During the execution of the patterns, we recorded the bilateral activation of the TAm in root mean square (RMS). We used the Student paired $t$-test for the first and last treatments, $P$-value set at $<0.05$. Clinical significance (CS) was obtained by subtracting the values of the first treatment from the last. Chopping pattern to the right side increased RMS for the right $(t=-3.52, C S=52 \%)$, but not the left TAm ( $t=$
-3.35). Flexion-abduction with external rotation pattern to the right $(t=$ $-2.46, C S=55 \%)$ and left $(t=-2.07, C S=53 \%)$ significantly increased RMS for TAm on both sides. Extension-adduction with internal rotation pattern to the right $(t=-0.25)$ and left $(t=-1.84)$ did not produce any changes in TAm. Ipsilateral PNF patterns selectively produce TAm overflow in peripheral polyneuropathy patients and can be successfully used as supportive therapy for foot drop.

Keywords: Proprioceptive neuromuscular facilitation, Overflow, Charcot-Marrie-Tooth disease, Foot drop

\section{INTRODUCTION}

Proprioceptive neuromuscular facilitation (PNF) works with the muscle and joint proprioceptors to facilitate neuromuscular system performance (Showman, 1962). PNF uses diagonal patterns of movement with maximal muscle strength, in isotonic and isometric muscle contractions, to achieve the desired objectives (Rhyu et al., 2015). To facilitate the contraction of the weak muscles, PNF uses force muscle irradiation, stimulating the strong muscles (Abreu et al., 2015; Gontijo et al., 2012; Hindle et al., 2012; Meningroni et al., 2009; Pink, 1981). Force muscle irradiation is the process where a stimulus reaches the spinal cord through the peripheral nervous system and then returns to the muscle (Ivanenko et al., 2004).

PNF is widely used for rehabilitation in patients with encephalic dysfunctions (Krukowska et al., 2016; Ribeiro et al., 2014), however there are very few studies in patients with peripheral nervous system injury (Meningroni et al., 2009).

Charcot-Marie-Tooth (CMT) is part of a heterogeneous group of clinical diseases and genetic disorders. CMT is a hereditary motor and sensory neuropathy that produces progressive muscle atrophy and weakness of the extremities, sensory loss and decreased or absent tendon reflexes (Reilly et al., 2011). This neuropathy can be classified into type 1 or 2 , but both are very similar, from a clinical standpoint. Type $1 \mathrm{~A}$ is the most common form of CMT (CMT-1A) and is the result of duplication on chromosome

\footnotetext{
${ }^{*}$ Corresponding author: João Eduardo de Araujo

(D) https://orcid.org/0000-0001-8709-318X

Laboratory of Neuropsychobiology and Motor Behavior, Department of Health Sciences, Ribeirão Preto Medical School of the University of São Paulo (USP), Av. dos Bandeirantes, 3900, Ribeirão Preto (SP) 14049-900, Brazil

Tel: +55-16-33150738, Fax: +55-16-33154413, E-mail: araujoje@fmrp.usp.br

Received: March 19, 2018 / Accepted: April 25, 2018
} 
17p11.2, with encoding myelin protein, which causes a decrease in conduction velocity of peripheral nerves and distal muscle strength (Krajewski et al., 2000). Muscle weakness affects various muscles in the lower limbs (LLs). One muscle with clinical importance, for the CMT-1A patients, is the tibialis anterior muscle (TAm). The TAm failure manifests clinically as "foot drop," and it is found in a high percentage of people affected by the disease (Sackley et al., 2009). These changes in muscle strength and ankle biomechanics can interfere with walking and contribute to foot deformities in this population (Burns et al., 2005).

Physical therapists utilize many approaches for foot drop rehabilitation, and PNF is one of them. We reported previously that PNF patterns produce overflow in patients with CMT-1A in contralateral upper limb (UL) and LL (Meningroni et al., 2009). Since it is possible to control TAm failure in CMT-1A, using contralateral overflow, it is essential to investigate other FNP patterns, to understand if they can produce overflow in patients with demyelinating polyneuropathy. Thus, this study analyzed ipsilateral PNF and its effects on TAm, after five weeks of treatment.

\section{MATERIALS AND METHODS}

\section{Participants}

We evaluated and treated 13 individuals of both sexes, with CMT-1A disease, aged 15 to 64 years, presenting muscle weakness and abnormal gait. All patients were recruited from the outpatient clinic of Neurogenetics, Department of Neuroscience and Behavioral Sciences of the Hospital of Ribeirão Preto Medical School, at the University of São Paulo (Hospital das Clínicas, HCFMRP/USP). The CMT-1A diagnosis was given by a neurologist, using family history, as well as electrophysiological and genetic testing. The subjects were in different stages of the disease, ranging from moderate to severe.

The inclusion criteria were: (a) preserved cognition, (b) no joint blockages (range of motion preserved), and (c) ability to walk, with or without assistance. The exclusion criteria were: (a) cardiac arrhythmias, (b) uncontrolled hypertension, and (c) severe cardiovascular and respiratory problems. The use of medications for other diseases was accepted. All participants signed an informed consent form, prior to the start of the study.

The Ethics in Human Research Committee of HCFMRP/USP (protocol n ${ }^{\circ}$ 10530/2007) approved this project. This methodology was registered in the National System of Information Ethics and Human Research (CAAE- 0445.0.004.000-07) to be appropriate, ethically and methodologically, as per the precepts of Reso-
Recruitment of thirteen individuals with CMT-1A disease

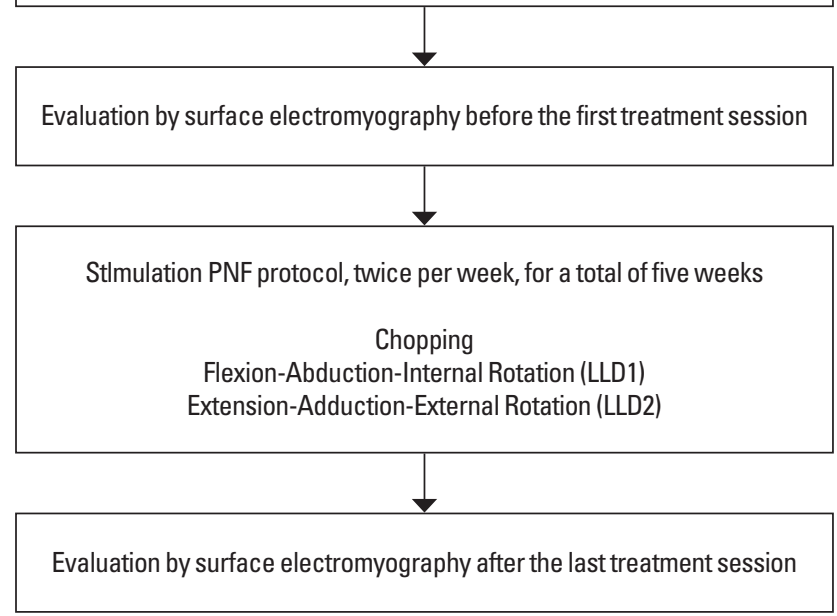

Fig. 1. Flowchart showing the subject selection, evaluations, and stimulation protocol. PNF, proprioceptive neuromuscular facilitation. CMT-IA, Charcot-Marie-Tooth disease.

lution 196/96 of the National Health Council.

\section{Study protocol}

The participants were assigned to one single treatment group (single-subject design study). The PNF patterns adopted in this study aimed to produce ipsilateral overflow in the TAm. Each patient's evaluation happened before the first and after the last treatment session (Fig. 1).

Physical therapy sessions using PNF were performed twice per week, for a total of five weeks and ten therapy sessions. The International Association of Proprioceptive Neuromuscular Facilitation certified the physiotherapist who was responsible for the PNF sessions. We use the following PNF patterns:

Chopping: The patients were seating on the stretcher with both shoulders in flexion, abduction and external rotation; elbows in extension and wrist and fingers in flexion. The patients shook the physical therapist's hand; and moved both UL simultaneously in the direction of the hip at left or at right, without flexing the elbow (Showman, 1962).

Flexion-abduction-internal rotation (LLD1): The patients were positioned in the supine position, lying down on the stretcher. One of the LL was positioned in extension, external rotation, and adduction. They were instructed to perform flexion, abduction, and internal rotation (Showman, 1962).

Extension-adduction-external rotation (LLD2): The patients were positioned in the supine position, lying down on the stretch- 
er. One of the LL was positioned in flexion, abduction and internal rotation of the hip. They were instructed to perform extension, adduction and external rotation (Showman, 1962).

All patterns of movement met resistance from the physical therapist's hands. The maximum resistance was used, that is, the resistance that was tolerated by each patient, and that allowed for the execution of the movement pattern in a complete range of motion. All patterns were repeated four times, with an average execution time of $6 \mathrm{sec}$. The patients individually determined the resting period between the patterns. Each session lasted an average of $40 \mathrm{~min}$. During the treatment, we respected the functionality, strength and muscular endurance of each patient. The same physical therapist applied all the patterns. Evaluations and assistance were provided at the Rehabilitation Center-HCFMRP/USP.

\section{Neurophysiologic measurement: electromyography}

Electromyography was performed by a 4-channel electromyography biofeedback equipment EMG-610C model, with a bandpass filter and a cutoff frequency between $20-500 \mathrm{~Hz}$, amplified with a 1,000 gain and common mode bounce rate $>120 \mathrm{~dB}$ (EMG System of Brazil, São José dos Campos, Brazil). We used a notebook HP Pavillion dv2000 brand, with AMD Turion 64X2. The equipment was connected to the battery, with no contact with the electrical network for stabilizing the electromyography (EMG) signal and filtering possible noises from the power supply.

A disposable surface electrode, double and bipolar $\mathrm{Ag} / \mathrm{AgCl}$ (silver/silver chloride), made with polyethylene hypoallergenic foam medical adhesive, was placed bilaterally on the TAm muscle belly, with a fixed interelectrode distance of $20 \mathrm{~mm}$. We used a disposable razor to remove the hair from the skin. An abrasion was performed using a nail file to remove dead skin cells, and the area was subsequently cleaned with $70 \%$ alcohol (Costa and de Araujo, 2008; de Souza et al., 2016; Zanin et al., 2014).

The EMG signal in root mean square (RMS) was obtained during the execution of the PNF patterns. Since each pattern had an average duration of $6 \mathrm{sec}$, we discarded the first and final seconds of each sampling. For data acquisition, DATAQ Instruments Hardware Manager software (DATAQ Instruments, Akron, $\mathrm{OH}$, USA) was used. All procedures mentioned above are in accordance with the recommendations of the European Union for surface electromyography noninvasive muscular project (SENIAM project, 2006).

All subjects performed active dorsiflexion at the beginning of the first session, with the right and left foot. The physical therapists verbally requested that the patient use the highest strength possible to perform the movement.

\section{Statistics}

The patterns were repeated 4 times for each side of the body, thus, we used the average of the four RMS values for each pattern ([RMS execution $1+$ RMS execution $2+$ RMS execution $3+$ RMS execution 4$] \div 4=$ RMS pattern). RMS data obtained in the first session were normalized and transformed into the percentage. Therefore, the average RMS for each diagonal, at the beginning of the treatment, corresponds to $100 \%$ of TAm activation. RMS data obtained in the last session were normalized as the percentage, taking into account the results of the first evaluation. The data (first and last session) showed variance and normality according to the Kolmogorov-Smirnov test, thus it was analyzed by Student paired $t$-test, the $P$-value was set at $<0.05$. The clinical significance was obtained by the subtraction of the values obtained in the last session by the values obtained in the first session. This data was presented in percentage.

\section{RESULTS}

Ipsilateral force overflow was produced by chopping pattern on the right side of the body, as RMS values in the TAm increased, when comparing the last session to the first $(t=-3.52, P<0.05)$. However, the same was not true for the patterns applied to the left side of the body $(t=-3.35, P=0: 21)$. In the LLD2 pattern, we did not find any changes in the TAm RMS values, for right or left ( $t=$ $-2.46, P=0.80$ and $t=-1.84, P=0: 10$, respectively). On the other hand, the ipsilateral LLD1 pattern produced overflow, as an increase in the TAm RMS values on the right and left sides ( $t=$ -2.46, $P<0.05$ and $t=-2.07, P<0.05$, respectively). Clinically, overflow obtained with the chopping pattern on the right was $52 \%$, and the LLD1 pattern was 55\% for the right and 53\% for the left side (Fig. 2).

\section{DISCUSSION}

The patients who participated in our study showed the inherent muscle weakness for TAm activation that is characteristic of the CMT-IA disease. This weakness is caused by the myelin sheath modification, which reduces the conduction speed on the peripheral nerves (Borg and Ericson-Gripenstedt, 2002). Despite the reduced speed conduction of the stimulus in the peripheral nerves, our results show that a short-term intervention program, based on the ipsilateral pattern of PNF, can improve the TAm RMS re- 

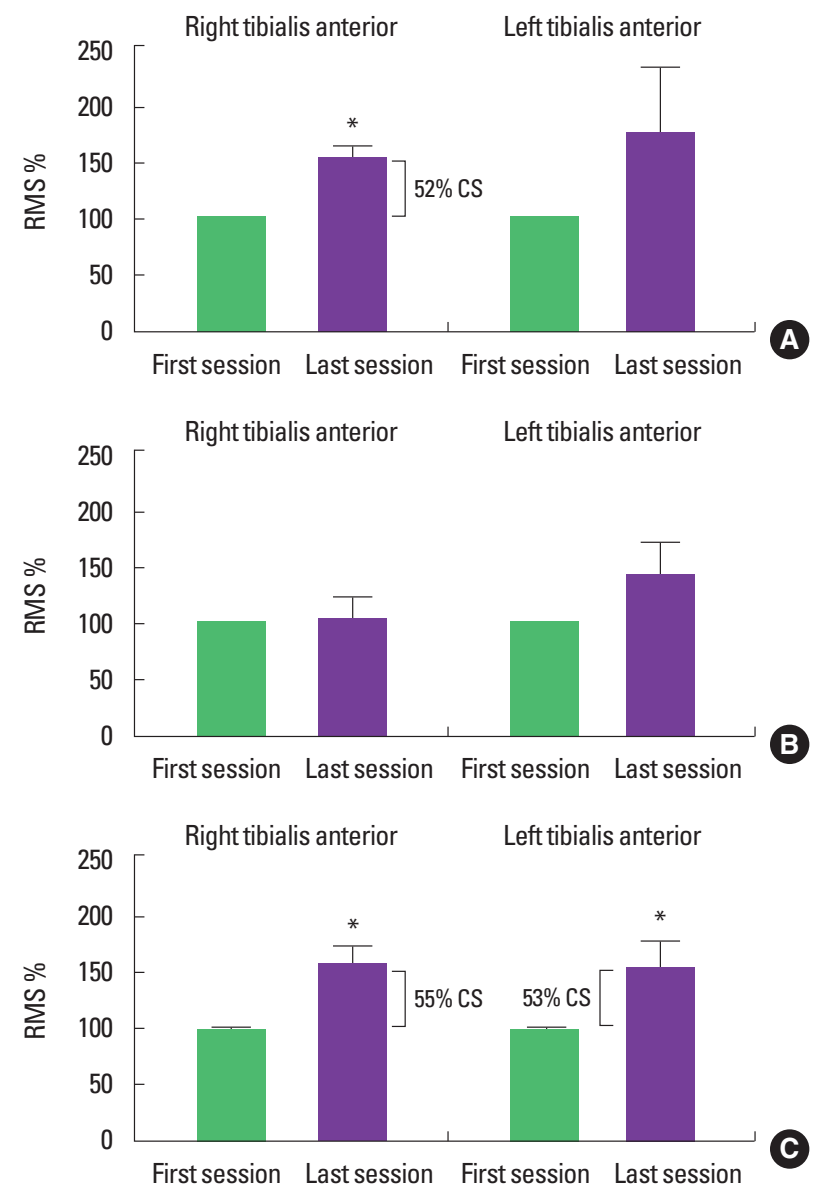

Fig. 2. Percentage of the root mean square (RMS) values on the left and right tibialis anterior muscle in the first (white columns) and last session (black columns). (A) Proprioceptive neuromuscular facilitation (PNF) chopping pattern. (B) PNF extension-adduction-external rotation pattern. (C) PNF flexion-abduction-internal rotation pattern. The columns represent the mean, and the bars represent + standard error of the mean. In panels $A$ and $C$, CS value is the clinical significance in $\%$. ${ }^{*} P>0.05$. The number of subjects $=13$.

sponse.

Rehabilitation treatments can offer possibilities to improve the muscle strength in CMT-1A patients. The progressive resistance-training program applied directly to the weak muscles (Chetlin et al., 2004) and the moderate intensity muscle training (Kilmer, 2002) seems to be adequate for these patients. In general, the effectiveness of these techniques can be attributed to an exercise of low duration that takes into account the individual response and the stage of the disease. This corroborates the wellknown fact that high-intensity exercises do not bring improvements to this population (Fowler, 2002).

Our PNF protocol used a short duration muscle contraction, with low repetition of patterns and longer resting time, as described by other authors (Chetlin et al., 2004; Kilmer, 2002). The patients can perform the patterns using the full range of motion and, despite the maximal muscle resistance, our protocol is characterized as moderate exercise. Furthermore, the exercises are performed by the strong muscle groups, and not by the weak muscle groups, that need to be treated, and this is highly relevant because it allows the patients to actively participate in the treatment.

Resistance exercises, in healthy subjects, can produce recruitment of other muscle groups as the synergists, improving the movement strength and producing contralateral overflow (Pink, 1981). Additionally, previous work from our research group showed that PNF contralateral patterns in CMT-1A patients increase the TAm activation (Meningroni et al., 2009). While studies with overflow show this effect in the contralateral side of the body, our results show the possibility of producing overflow on the same side of the body (Abreu et al., 2015; Gontijo et al., 2012).

Studies in the literature have consistently shown that older adults use homologous muscles contralaterally, during voluntary contractions (Bodwell et al., 2003; Shinohara et al., 2003). This involuntary muscle response changes the corticospinal output (Gross et al., 2005) and modulates the corticomuscular coherence (Johnson et al., 2011). This concept is fundamental to our results, because if CMT-1A does not allow for peripheral nerve improvement, then the effect that we showed in the present study is related to a central nervous system adaptation. It is known that neuromuscular adaptation occurs from resistance exercises, which changes the way the muscles are recruited by the central nervous system, allowing for motor learning and better muscle recruitment (Carroll et al., 2001).

Despite the possible corticospinal effect, the PNF TAm activation seen here is likely related to spinal circuits involved with control of rhythmic locomotion (Guertin, 2013). While flexion of the trunk, hip, and knee produced flexion of the hip; LL extension produces ankle extension that facilitates plantar flexion movement. This is most likely why chopping and LLD1 improved the TAm activation, while LLD2 did not. Thus, it is extremely important to understand the biomechanical pattern of body movement for producing a substantial overflow.

The present results are consistent with the results from our previous study (Meningroni et al., 2009) using PNF contralateral patterns which activated TAm in CMT-1A. Our results show that the ipsilateral patterns of FNP can be a reasonable and helpful treatment for foot disorders in this population. The TAm effect is present while the treatment is in progress.

The present study is limited by the lack of follow up after the 
treatment due to the need for travelling far to the HCFMRP/USP Hospital to participate. Thus, there is need for further studies, to follow these patients for a more extended period, to see if the TAm effects remain.

\section{CONFLICT OF INTEREST}

No potential conflict of interest relevant to this article was reported.

\section{ACKNOWLEDGMENTS}

This work was funding by the Brazilian National Council for Scientific and Technological Development- CNPq (process 408861/2006-1). Carolina S. Nakada and Paula C. Meningroni was the recipient of a scientific initiation scholarship from FAPESP (process 2007/05803-3 and 2007/03285-5). Amanda C. Fuzaro was the recipient of a doctoral scholarship from FAPESP (process 2011/10017-2).

\section{REFERENCES}

Abreu R, Lopes AA, Sousa AS, Pereira S, Castro MP. Force irradiation effects during upper limb diagonal exercises on contralateral muscle activation. J Electromyogr Kinesiol 2015;25:292-297.

Bodwell JA, Mahurin RK, Waddle S, Price R, Cramer SC. Age and features of movement influence motor overflow. J Am Geriatr Soc 2003;51:17351739.

Borg K, Ericson-Gripenstedt U. Muscle biopsy abnormalities differ between Charcot-Marie-Tooth type 1 and 2: reflect different pathophysiology? Exerc Sport Sci Rev 2002;30:4-7.

Burns J, Crosbie J, Hunt A, Ouvrier R. The effect of pes cavus on foot pain and plantar pressure. Clin Biomech (Bristol, Avon) 2005;20:877-882.

Carroll TJ, Riek S, Carson RG. Neural adaptations to resistance training: implications for movement control. Sports Med 2001;31:829-840.

Chetlin RD, Gutmann L, Tarnopolsky M, Ullrich IH, Yeater RA. Resistance training effectiveness in patients with Charcot-Marie-Tooth disease: recommendations for exercise prescription. Arch Phys Med Rehabil 2004;85:1217-1223.

Costa LA, de Araujo JE. The immediate effects of local and adjacent acupuncture on the tibialis anterior muscle: a human study. Chin Med 2008:3:17.

de Souza LL, de Araujo FL, da Silva FA, Mucciaroni TS, de Araujo JE. Unilateral and immediate stimulation of acupuncture points Xiaohai (SI8) and Jianwaishu (SI14) of the small intestine meridian increases electromyographic activity and strength in the ipsilateral and contralateral upper trapezius muscle. J Acupunct Meridian Stud 2016;9:250256.

Fowler WM Jr. Role of physical activity and exercise training in neuromuscular diseases. Am J Phys Med Rehabil 2002;81(11 Suppl):S187195.

Gontijo LB, Pereira PD, Neves CD, Santos AP, Machado Dde C, Bastos $\mathrm{VH}$. Evaluation of strength and irradiated movement pattern resulting from trunk motions of the proprioceptive neuromuscular facilitation. Rehabil Res Pract 2012;2012:281937.

Gross J, Pollok B, Dirks M, Timmermann L, Butz M, Schnitzler A. Taskdependent oscillations during unimanual and bimanual movements in the human primary motor cortex and SMA studied with magnetoencephalography. Neuroimage 2005;26:91-98.

Guertin PA. Central pattern generator for locomotion: anatomical, physiological, and pathophysiological considerations. Front Neurol 2013;3: 183.

Hindle KB, Whitcomb TJ, Briggs WO, Hong J. Proprioceptive neuromuscular facilitation (PNF): Its mechanisms and effects on range of motion and muscular function. J Hum Kinet 2012;31:105-113.

Ivanenko YP, Poppele RE, Lacquaniti F. Five basic muscle activation patterns account for muscle activity during human locomotion. J Physiol 2004;556(Pt 1):267-282.

Johnson AN, Wheaton LA, Shinohara M. Attenuation of corticomuscular coherence with additional motor or non-motor task. Clin Neurophysiol 2011;122:356-363.

Kilmer DD. Response to resistive strengthening exercise training in humans with neuromuscular disease. Am J Phys Med Rehabil 2002;81(11 Suppl):S121-126.

Krajewski KM, Lewis RA, Fuerst DR, Turansky C, Hinderer SR, Garbern J, Kamholz J, Shy ME. Neurological dysfunction and axonal degeneration in Charcot-Marie-Tooth disease type 1A. Brain 2000;123 (Pt 7): 1516-1527.

Krukowska J, Bugajski M, Sienkiewicz M, Czernicki J. The influence of NDT-Bobath and PNF methods on the field support and total path length measure foot pressure (COP) in patients after stroke. Neurol Neurochir Pol 2016;50:449-454.

Meningroni PC, Nakada CS, Hata L, Fuzzaro AC, Marques W Jr, Araujo JE. Contralateral force irradiation for the activation of tibialis anterior muscle in carriers of Charcot-Marie-Tooth disease: effect of PNF intervention program. Braz J Phys Ther 2009;13:438-443.

Pink M. Contralateral effects of upper extremity proprioceptive neuromuscular facilitation patterns. Phys Ther 1981;61:1158-1162.

Reilly MM, Murphy SM, Laurá M. Charcot-Marie-Tooth disease. J Peripher Nerv Syst 2011;16:1-14. 
Rhyu HS, Kim SH, Park HS. The effects of band exercise using proprioceptive neuromuscular facilitation on muscular strength in lower extremity. J Exerc Rehabil 2015;11:36-40.

Ribeiro TS, de Sousa e Silva EM, Sousa Silva WH, de Alencar Caldas VV, Silva DL, Costa Cavalcanti FA, Lindquist AR. Effects of a training program based on the proprioceptive neuromuscular facilitation method on post-stroke motor recovery: a preliminary study. J Bodyw Mov Ther 2014;18:526-532

Sackley C, Disler PB, Turner-Stokes L, Wade DT, Brittle N, Hoppitt T. Rehabilitation interventions for foot drop in neuromuscular disease. Cochrane Database Syst Rev 2009;(3):CD003908.
SENIAM project. Surface ElectroMyoGraphy for the non-invasive assessment of muscles. Enschede (the Netherlands): SENIAM; 2006 [cited 2007 Oct 4]. Available from: http://www.seniam.org.

Shinohara M, Keenan KG, Enoka RM. Contralateral activity in a homologous hand muscle during voluntary contractions is greater in old adults. J Appl Physiol (1985) 2003;94:966-974.

Showman JC. The rationale of patterns and techniques of proprioceptive neuromuscular facilitation. Aust J Physiother 1962;8:115-120.

Zanin MS, Ronchi JM, Silva Tde C, Fuzaro AC, Araujo JE. Electromyographic and strength analyses of activation patterns of the wrist flexor muscles after acupuncture. J Acupunct Meridian Stud 2014;7:231-237. 